\title{
Association of the 3' Region of COMT with Schizophrenia in Taiwan
}

\author{
Yi-Ling Chien, ${ }^{1,2}$ Chih-Min Liu, ${ }^{2,3}$ Cathy Shen-Jang Fann, ${ }^{4}$ Yu-Li Liu, ${ }^{5}$ Hai-Gwo Hwu $u^{3,6 *}$
}

Background/Purpose: The Val ${ }^{108 / 158}$ Met (rs4680) single nucleotide polymorphism (SNP) in the catecholO-methyltransferase (COMT) gene contributes to genetic susceptibility to schizophrenia, which is specifically related to impairments in executive functioning. A different genomic region composed of three SNPs (rs737865, rs4680, rs165599) within the COMT gene has been reported to be significantly associated with schizophrenia in Ashkenazi Jews. This study aims to clarify the association between these three SNPs and their haplotypes with schizophrenia and neurocognitive functioning, using both case-control and family-based designs.

Methods: The case-control study included 124 schizophrenia patients and 112 healthy controls, while the family samples included 83 families with at least two affected siblings. The neurocognitive functioning was assessed by the Continuous Performance Test (CPT) and Wisconsin Card Sorting Test. The association analysis was performed using TRANSMIT and FBAT.

Results: There was no significant association between the three SNPs and schizophrenia in the case-control study. In the family study, the A allele of rs 165599 was transmitted preferentially to the affected individuals $(p=0.023)$, and significantly associated with a later age of onset $(p=0.018)$, more severe delusion/ hallucination symptom dimension $(p=0.027)$, and poorer performance in the CPT ( $p=0.04)$. The triple SNP haplotypes did not reveal any significant association with schizophrenia or neurocognitive function. Conclusion: The SNP rs165599, which has been mapped to the 3'-UTR region of the COMT gene, was significantly associated with schizophrenia in our family study, and possibly associated with the age of onset, delusion/hallucination symptom dimension, and CPT performance. Therefore, COMT may contribute to the genetic risk for schizophrenia not through the $\mathrm{Val}^{108 / 158} \mathrm{Met}$ polymorphism, but through other variants that are situated 3' to this region, in the Taiwanese population. Nevertheless, the true associated functional variants in our subjects remain to be elucidated. [J Formos Med Assoc 2009;108(4):301-309]

Key Words: case-control studies, catechol O-methyltransferase, family-based association study, schizophrenia

It is estimated that $65-70 \%$ of the susceptibility to schizophrenia can be attributed to a genetic component. ${ }^{1}$ Even though schizophrenia is highly heritable, genetic linkage studies have failed to establish a common chromosomal region linked with this disorder. This suggests that multiple genes combine to cause the disorder and that each factor confers only a small degree of risk for the disease. ${ }^{2}$

Multiple lines of evidence implicate dopaminergic system dysfunction in the pathogenesis

(C)2009 Elsevier \& Formosan Medical Association

${ }^{1}$ Department of Psychiatry, National Taiwan University Hospital, Yun Lin Branch, Yunlin; ${ }^{2}$ Graduate Institute of Clinical Medicine, National Taiwan University College of Medicine, ${ }^{3}$ Department of Psychiatry, National Taiwan University Hospital and National Taiwan University College of Medicine, and ${ }^{4}$ Institute of Biomedical Science, Academia Sinica, Taipei; ${ }^{5}$ Division of Mental Health and Substance Abuse Research, National Health Research Institutes, Miaoli; ${ }^{6}$ Institute of Epidemiology, College of Public Health, National Taiwan University, Taipei, Taiwan.

Received: September 8, 2008

Revised: September 9, 2008

ELSEVIER Accepted: September 9, 2008
* Correspondence to: Dr Hai-Gwo Hwu, Department of Psychiatry, National Taiwan University Hospital, 7 Chung-Shan South Road, Taipei 100, Taiwan.

E-mail: haigohwu@ntu.edu.tw 
of schizophrenia. Therefore, the catechol-Omethyltransferase (COMT) gene, which codes for a principle enzyme that catalyzes the metabolism of dopamine, is considered as one of the candidate genes that contribute to the risk for schizophrenia. Furthermore, the micro-deletion on chromosome 22q11 region, which includes the COMT gene and causes velocardiofacial syndrome, is associated with a high rate of psychosis, particularly schizophrenia. ${ }^{3}$

The most commonly examined locus within the COMT gene is a functional polymorphism at codon 158 of the membrane-bound form of the enzyme ( $\left.\mathrm{Val}^{108 / 158} \mathrm{Met}\right)$. The enzyme activity decreases to one quarter of the wild-type level in the substituted form, which results from a G to A transition at the single nucleotide polymorphism (SNP) rs4680. In addition, the valine variation has been shown to correlate with poorer executive functioning and prefrontal cortical activity, specifically on the Wisconsin Card Sorting Test $(\mathrm{WCST})^{4,5}$ and the N-Back Working Memory Task. ${ }^{5-7}$ However, not all association studies that have examined COMT as a candidate gene for schizophrenia have shown a positive association. Two recent meta-analyses of case-control studies did not support this association in European and Asian populations. ${ }^{8,9}$

Shifman et $\mathrm{al}^{10}$ found a unique haplotype (G-G-G haplotype of the three SNPs, rs737865, rs4680 and rs165599) of COMT that is highly associated with schizophrenia in Ashkenazi Jews. This haplotype has also been reported to be linked with lower expression of COMT mRNA in the human brain. ${ }^{11,12}$ Two independent studies have reported a significant association with different haplotypes of a triple SNP haplotype block. ${ }^{13,14}$ These findings imply that the genetic association between COMT and schizophrenia probably result from SNPs that occur in several regions of the gene.

Previous studies of Asian populations using case-control designs or trio samples have not supported the association of SNP rs4680 with schizophrenia. ${ }^{9,15-19}$ However, other COMT polymorphisms have been less well studied using
Asian family studies. Moreover, the association between cognitive function and the COMT genotype in Asian schizophrenia patients remains unclear. Hence, the present study aimed to clarify the association of three SNPs (rs737865, rs4680, rs165599) and their haplotypes with schizophrenia and neurocognitive functioning, as assessed by the Continuous Performance Test (CPT) and WCST, using case-control and family-based association studies.

\section{Methods}

\section{Subjects}

The schizophrenia patients in the case-control study were recruited from patients consecutively admitted to the acute inpatient psychiatric wards of the Department of Psychiatry, National Taiwan University Hospital and the university-affiliated hospitals of Taipei City Psychiatric Center and Taoyuan Psychiatric Center. All patients fulfilled the diagnostic criteria of schizophrenia according to DSM-IV definitions. Patients with major physical and neurological illness or substance use disorder were excluded. After informed consent was obtained, the study subjects received a clinical interview for comprehensive assessment of clinical features, and cross-sectional psychopathology examination using the Positive and Negative Syndrome Scale (PANSS) ${ }^{20}$ when they were stabilized after treatment and prepared for discharge. In addition, the course of illness during index admission was recorded and whole blood was collected. A total of 124 patients were recruited in this study, and 91 received the CPT and 87 the WCST at the same time as clinical rating. A total of 112 normal controls without a history of psychiatric disorders were recruited, mostly from hospital staff.

Schizophrenia probands who had at least two affected siblings were identified from the outpatient clinics of the Department of Psychiatry, National Taiwan University Hospital and the university-affiliated Taoyuan Psychiatric Center. Data collection was initiated after informed 
consent was obtained from the identified study subjects and their families. All of the family members were personally interviewed by the research psychiatrists using the Psychiatrist Diagnostic Assessment (PDA) ${ }^{21}$ Clinical symptoms were rated using the Schedule for Assessment of Negative Symptoms (SANS) 22 and the Schedule for Assessment of Positive Symptoms (SAPS), ${ }^{23}$ with satisfactory reliability. The final diagnostic assessment was formulated by integrating the PDA data and the clinical information from the medical chart records. The final diagnosis was performed according to DSM-IV criteria. The family samples included 324 individuals with DNA available from 83 families with at least two affected siblings, and 118 individuals received the СРT and 103 the WCST at the same time as clinical rating.

The demographic data for the case-control study was $57 \%$ male cases and $38 \%$ male controls, and the mean age was $37.5 \pm 6.8$ years for the cases and $31.8 \pm 7.6$ for the controls. The mean age of onset was $21.9 \pm 5.5$ years. In the family study, there were $62 \%$ male subjects among the affected individuals, with a mean age of $32.7 \pm 7.7$ years, and the mean age of onset was $22.4 \pm 6.3$ years. The founders (i.e. parents of the probands) were $45 \%$ male, with a mean age of $60.2 \pm 8.1$ years.

The clinical ratings of schizophrenia subjects according to neuropsychological tests were as follows. In the case-control study, the mean score of the positive symptom subscale of PANSS was $1.95 \pm 0.68$, negative symptom subscale was $2.45 \pm 0.93$, and the general psychopathology subscale was $1.76 \pm 0.48$. In the family study, the mean score for the delusion/hallucination symptom dimension of SAPS and SANS was $1.34 \pm 1.38$, the negative symptom dimension was $1.73 \pm 1.53$, and the disorganization symptom dimension was $1.16 \pm 1.32$. The subjects in both samples were in a relatively stable state.

\section{CPT}

A CPT machine from Sunrise Systems, v. 2.20 (Pembroke, MA, USA), was used to assess sustained attention. The procedure has been described in detail elsewhere. ${ }^{24}$ Briefly, numbers from zero to nine were randomly presented for 50 milliseconds, each at a rate of one per second. Each subject underwent two different CPT sessions that included the undegraded 1-9 task and the 25\% degraded 1-9 task. Subjects were asked to respond whenever the number nine was preceded by the number one on the screen. During the $25 \%$ degraded session, a pattern of snow was used to toggle the background and foreground in order to distort the image. Sensitivity (d') was derived from the hit rate (probability of a response to target trials) and false-alarm rate (probability of response to non-target trials). ${ }^{25}$ Sensitivity is an individual's ability to discriminate target from non-target stimuli. The effect of age, education and sex on CPT performance was adjusted, based on a community sample of 345 subjects. ${ }^{24}$ CPT performance was calculated from the $\mathrm{z}$ scores of the undegraded and degraded CPT results, which were adjusted for these demographic features. Subsequently, the $\mathrm{z}$ scores of these performance indicators were used for further study analyses.

\section{WCST}

We employed a computerized version of the WCST $^{26}$ that had been applied in a previous study of the Taiwanese population. ${ }^{27}$ During the test, subjects were required to match 128 response cards to the four stimulus cards along one of three dimensions (color, form, number), by pressing one of the four number keys on the computer keyboard. Subjects were not informed of the correct sorting principle, nor were they told when the principle would shift during the test, but they were given feedback ("right" or "wrong") on the screen after each trial. The indexes of WCST $^{28}$ used for this study were the following: (1) perseverative errors, which reflected the tendency towards perseveration; and (2) categories achieved, which was the number of times that 10 consecutive correct responses were made, which reflected overall success. These indicators were found to be impaired in schizophrenia probands ${ }^{29,30}$ and in their first-degree relatives. ${ }^{31}$ 
The effect of age, education and sex on WCST performance was adjusted based on 211 healthy screened controls (unpublished data from ShengHsiang Lin, Chih-Min Liu, Shi-Kai Liu, Institute of Epidemiology, College of Public Health, National Taiwan University, Taipei, Taiwan, 2008). WCST performance was calculated as the $\mathrm{z}$ scores of the perseverative errors and category achieved, which was adjusted for these demographic features. Subsequently, the $\mathrm{z}$ scores of these performance indicators were used for further study analyses.

\section{Genotyping}

The three SNPs (rs737865, rs4680, rs165599) were genotyped using a PCR-RFLP method. The primers were designed using Primer 3.0 (http:// frodo.wi.mit.edu/cgi-bin/primer 3/primer 3-www. cgi). Briefly, PCR reactions were performed with 10 ng genomic DNA, $0.5 \mu \mathrm{L}$ of each PCR primer, $2.5 \mathrm{mM}$ dNTP, and $2.5 \mathrm{U}$ ProTaq DNA polymerase (Protech Technology Enterprise Co., Taipei, Taiwan). Thermal cycling conditions for PCR were as follows: $94^{\circ} \mathrm{C}$ for 2 minutes, followed by 35 cycles of $94^{\circ} \mathrm{C}$ for 45 seconds, $55^{\circ} \mathrm{C}$ for 30 seconds, and $72^{\circ} \mathrm{C}$ for 1 minute; followed by final extension at $72^{\circ} \mathrm{C}$ for 5 minutes. PCR products were treated by the restriction enzymes Bsc4I, NlaIII and HpaII for the rs737865, rs4680 and rs165599 genotyping, respectively. Digested products were then subjected to electrophoresis on an agarose gel, which was stained with ethidium bromide and the bands were visualized under ultraviolet light. DNA bands were read as follows: for rs737865, a 245-bp band indicated uncut and allele A, and a 163-bp band indicated cut and allele G; for rs4680, a 114-bp band indicated uncut and allele $\mathrm{G}$, and a 96-bp band indicated cut and allele A; for rs165599, a 242-bp band indicated uncut and allele A, and a 115-bp band indicated cut and allele G.

\section{Statistical analysis}

In order to verify the sample accuracy, including family relationship and genotype, PEDCHECK version $1.1^{32}$ and UNKNOWN version $5.23^{33}$ were used to check the patterns of Mendelian inheritance, and the procedure ALLELE of SAS/ GENETICS version 8.2 was used to test for the Hardy-Weinberg equilibrium. The allele and genotype frequency were calculated and compared by a $\chi^{2}$ test in the case-control study. Single loci and haplotype associations were tested by TRANSMIT $^{34}$ and FBAT. ${ }^{35}$ The association between SNP genotype and neuropsychological function was tested by the Kruskal-Wallis test in the casecontrol study and by haplotype FBAT ${ }^{35}$ in the family study. Linkage disequilibrium ( $\left.\mathrm{D}^{\prime}\right)$ was calculated with the Gold program. ${ }^{36}$

\section{Results}

All three SNPs tested in this study (rs737865, rs4680, rs165599) were compatible with the Hardy-Weinberg equilibrium in the case-control and family studies.

\section{Case-control study}

In the case-control study, G allele frequency among the cases was 0.29 for the SNP rs737865, 0.76 for rs 4680 , and 0.45 for rs 165599 . This was compared to controls with a $\mathrm{G}$ allele frequency of 0.25 for SNP rs737865, 0.75 for rs 4680 , and 0.48 for rs 165599 . There was no significant difference between the cases and controls for allele or genotype comparisons.

\section{Family study}

In the family study, the founder $\mathrm{G}$ allele frequency was 0.24 for the SNP rs737865, 0.79 for rs 4680 , and 0.48 for rs 165599 . The A allele of rs 165599 was transmitted preferentially to affected individuals, shown by different statistical methods, as demonstrated by TRANSMIT ( $p=0.023)$ and FBAT $(p=0.039)$. The detailed results are listed in Table 1 .

\section{Haplotype association analysis}

The $\mathrm{D}^{\prime}$ values of two SNPs are listed in Table 2. All the $\mathrm{D}^{\prime}$ values were lower than 0.7. Haplotype association analysis is shown in Table 3 . The triple SNP haplotype did not reveal a significant association with schizophrenia as shown by 


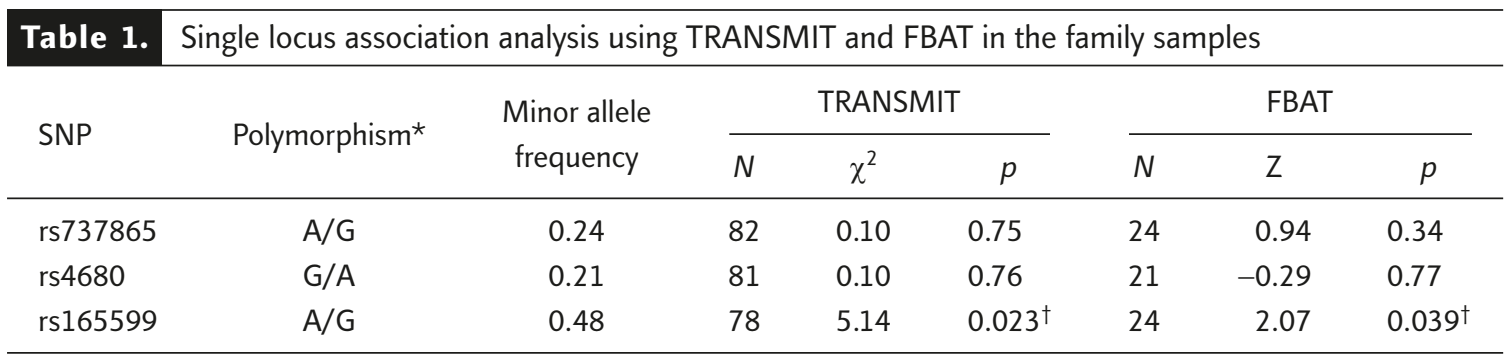

${ }^{*}$ Second allele under oblique line $(/)$ is the minor allele; ${ }^{\dagger}$ A allele is the over-transmitted allele. SNP $=$ single nucleotide polymorphism.

Table 2. Linkage disequilibrium ( $\left.\mathrm{D}^{\prime}\right)$ between the two studies' single nucleotide polymorphisms

\begin{tabular}{|c|c|c|c|c|c|c|}
\hline & \multicolumn{3}{|c|}{ Case-control study } & \multicolumn{3}{|c|}{ Family study } \\
\hline & rs737865 & rs4680 & rs165599 & rs737865 & rs4680 & rs165599 \\
\hline rs737865 & $\ldots$ & & & $\ldots$ & & \\
\hline rs4680 & 0.470 & $\ldots$ & & 0.100 & $\ldots$ & \\
\hline rs165599 & 0.471 & 0.652 & $\ldots$ & 0.253 & 0.414 & $\ldots$ \\
\hline
\end{tabular}

\begin{tabular}{cccc} 
Table 3. & \multicolumn{3}{l}{$\begin{array}{l}\text { Haplotype association analysis using } \\
\text { TRANSMIT }\end{array}$} \\
\hline Haplotype & HF & $\chi^{2}$ & $p$ \\
\hline A-G-A & 0.22 & 0.01 & 0.92 \\
G-G-A & 0.12 & 1.71 & 0.19 \\
A-A-A & 0.15 & 0.68 & 0.41 \\
G-A-A & 0.04 & 0.03 & 0.86 \\
A-G-G & 0.37 & 1.96 & 0.16 \\
G-G-G & 0.07 & 0.14 & 0.71 \\
A-A-G & 0.03 & 0.40 & 0.53 \\
G-A-G & 0.003 & 0.11 & 0.74 \\
\hline
\end{tabular}

$H F=$ haplotype frequency.

different statistical methods. The highly significant haplotype G-G-G in Ashkenazi Jews, ${ }^{10}$ and the A-G-A polymorphisms in an Irish study of high-density schizophrenia families ${ }^{13}$ were not significantly associated with schizophrenia in our studies.

\section{Phenotype analysis}

In the case-control study, the three SNPs and their haplotypes were not associated with the symptom profiles, age of onset, or CPT and WCST performance. In the family study, the A allele of SNP rs165599 was significantly associated with poorer performance in the degraded СРТ $(p=0.04)$, with a later age of onset $(p=0.018)$, and with a more severe delusion/hallucination symptom dimension $(p=0.027)$, which was calculated by the sum of the global scores for Delusion and Hallucination in the SAPS. The other two SNPs and all the triple SNP haplotypes were not associated with age of onset, symptom profiles, or CPT and WCST performance. The details are listed in Table 4.

\section{Discussion}

Our case-control study revealed no association between the three SNPs in COMT and schizophrenia. We found that the A allele of the SNP rs165599, which was located in the 3'-UTR region of the COMT gene, was significantly overtransmitted to the affected individuals in our family study, and was significantly associated with a later age of onset, more severe delusion/ hallucination symptom dimension, and poorer performance for the degraded CPT. In addition, the triple SNPs haplotypes were not significantly associated with schizophrenia in our subjects.

The functional polymorphism of $\mathrm{Val}^{108 / 158} \mathrm{Met}$ (rs4680) was not associated with schizophrenia in either our case-control or family study. These results were compatible with previous case-control 
Table 4. Phenotype analysis of three single nucleotide polymorphisms using haplotype FBAT in the family study

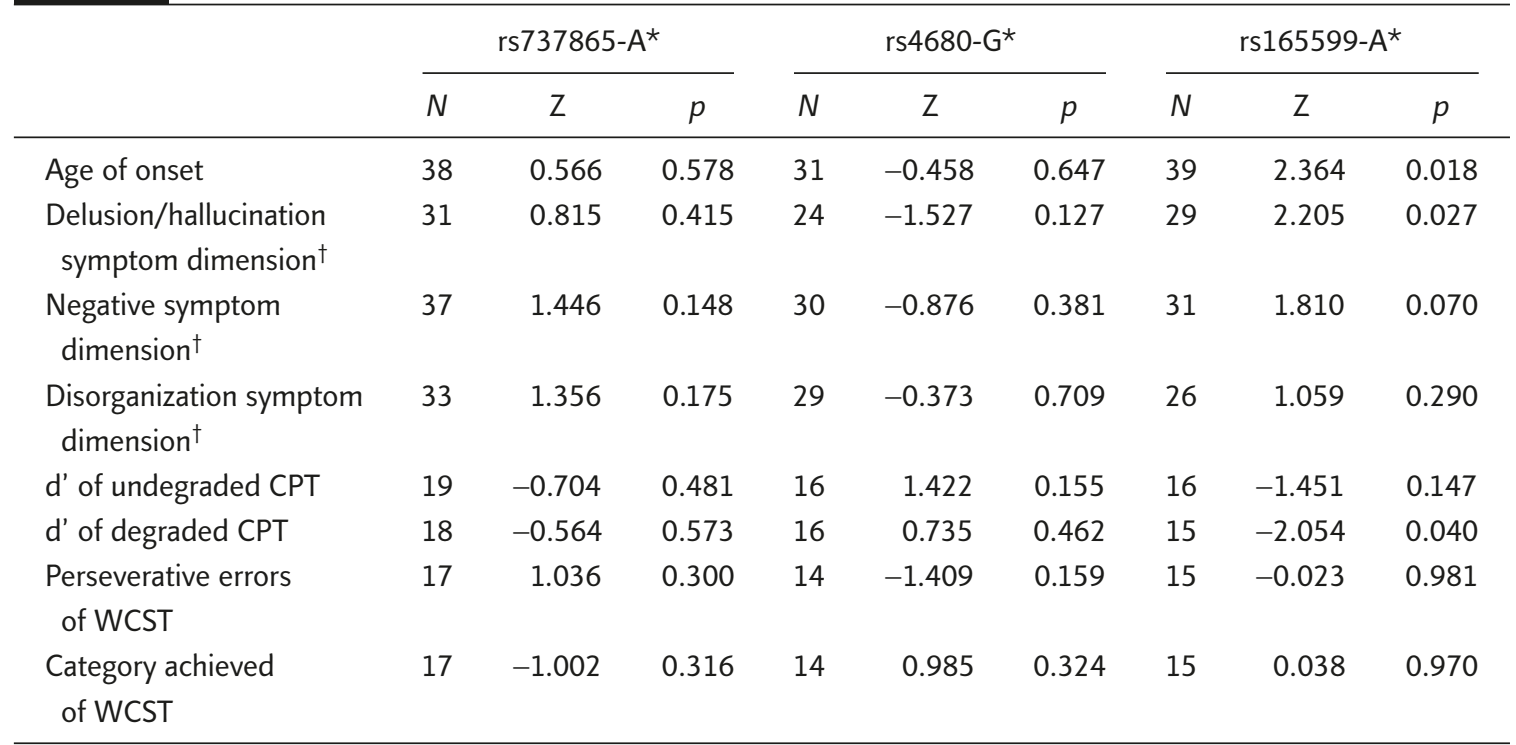

${ }^{*}$ Major allele of the SNP; ${ }^{+}$the delusion/hallucination symptom dimension was calculated by the sum of global scores of Delusions and Hallucinations in the SAPS; the disorganization symptom dimension was calculated by the sum of global scores of Positive Formal Thought Disorder and Bizarre Behavior in the SAPS; the negative symptom dimension was calculated by the sum of global scores of Affective Blunting, Alogia, Avolition-Apathy, and Anhedonia-Asociality in the SANS. N= family number.

studies of Asian subjects. ${ }^{9,16,17,19,37-39}$ Interestingly, the allele frequencies of this polymorphism were similar among most Asian subjects and very different from those in European subjects. ${ }^{9,40}$

We reported no significant association with the triple SNP haplotypes for the case-control or family study, which was consistent with the negative results previously reported in case-control studies of Asian populations. ${ }^{17,39,41}$ Among studies of European subjects, different populations have been reported to exhibit an association between schizophrenia and the different triple SNP haplotypes. Examples of this include the G-G-G haplotype in Ashkenazi Jews, ${ }^{10}$ the A-G-A haplotype in Irish families, ${ }^{13}$ and the A-A-G haplotype in Australian family samples. ${ }^{14}$ The $\mathrm{D}^{\prime}$ values among the three SNPs in our study were much lower than those in the European subjects and much closer to those of the Chinese Han subjects. ${ }^{39}$ The failure to replicate the triple SNP haplotype association in the Han population might reflect a differential linkage disequilibrium structure for the COMT gene, which arises from ethnic differences.

In this study, we found that SNP rs165599, but not the functional variant rs 4680 or the triple
SNP haplotypes, was associated with a diagnosis of schizophrenia, delusion/hallucination symptom dimension, age of onset, and CPT performance. This finding was different from other case-control association studies in Asian populations. ${ }^{17,39,41}$ Furthermore, the association with rs165599 was shown exclusively in our family study, but not in our case-control study, which implied that this association might be specific to the samples with higher genetic loading. The function of SNP rs165599 has been explored in several studies. Bray et $\mathrm{al}^{11}$ demonstrated that COMT is subject to allelic differences in expression level in the human brain. Specifically, the 3' flanking region of SNP rs165599 exhibited significant differences in allelic expression, with lower relative expression if the $G$ allele is at rs165599. This implies that the A allele at rs165599 in schizophrenia patients is associated with higher expression of the COMT gene, which is compatible with Egan et al's hypothesis of hypofrontality in schizophrenia. ${ }^{4}$ It has been reported that rs4680 interacts with SNP rs2097603 at the P2 promoter region and SNP rs165599 in the 3' region. These interactions have been implicated 
in predicting an inefficient prefrontal working memory response during a working memory paradigm in healthy control subjects. ${ }^{7}$ Recently, there have been numerous studies that have focused on the role of RNAi, which demonstrates that the 3' end of RNA transcripts may play a role in regulation of the transcriptomes. Whether rs165599 itself, or other functional variants in tight linkage disequilibrium with this SNP, contributes to the association demonstrated in the present study needs to be further elucidated.

An association between the functional SNP rs4680 and neurocognitive functioning has been reported in several studies, including with the WCST, ${ }^{5,42}$ Letter Number Sequencing, ${ }^{42} \mathrm{~N}$ back, $^{6,7}$ and performance on the attention domain of a neurocognitive battery. ${ }^{43}$ However, our study failed to replicate the previously observed association of this polymorphism with WCST and CPT performance. In addition, there have been few studies that have explored the association between the other SNPs within the COMT gene and neurocognitive function. Chan et al reported that SNP rs165599 is associated with visual reproduction, Stroop test, and 2-back reaction time for correct responses, but not with СРT in a relatively small Chinese sample. ${ }^{44}$ Our study is believed to be the first to show that the 3 ' variant of COMT may be associated with CPT performance.

The effects of the rs4680 genotypes on clinical phenotypes have been inconsistent across different studies. One study has reported an association with more negative symptoms and a younger age of onset, ${ }^{45}$ but several others have reported no evidence of such an association. ${ }^{37,46}$ The association of rs165599 with clinical phenotype has been less well studied previously. Our family study is believed to be the first to show that SNP rs 165599 is associated significantly with a later age of onset, and a more severe delusion/ hallucination symptom dimension. The association with delusion/hallucination symptom dimension in our family study seemed contradictory to the association between COMT and hypofrontality reported by Egan et $\mathrm{al}^{4}{ }^{4}$ which implied a lower function of dopamine in the prefrontal lobe in schizophrenia. On one hand, it has been found that the subcortical hyperdopaminergic function, which is considered to contribute to positive symptoms, is secondary to the prefrontal hypodopaminergic function. ${ }^{47}$ On the other hand, we observed that rs165599 is associated with the negative symptom dimension, with borderline significance $(p=0.07)$ (Table 4$)$. This was still consistent with the association between COMT and hypofrontality. However, this seems to conflict with the association of SNP rs165599 with schizophrenia with higher genetic loadings and later age of onset. Some studies have suggested that high familial loading is associated with early onset of schizophrenia. ${ }^{48}$ However, ascertainment bias ${ }^{49}$ and heterogeneity in age of onset have also been reported. ${ }^{50}$ Whether higher familial loading is associated with earlier onset of schizophrenia remains inconclusive. Also, there was no significant difference in age of onset between our family study $(22.4 \pm 6.3$ years $)$ and case-control study (21.9 \pm 5.5 years).

Although a positive association was found in the family study, the results should be interpreted with caution, and we cannot exclude the possibility of false-positivity or multiple-testing problems. Moreover, the sample size was relatively small and the power may have been inadequate for detecting this small genetic effect. Another limitation is that we did not adjust for the clinical factors, such as age of onset, when analyzing the genetic effects on neurocognitive function. Nevertheless, our findings implied that the COMT gene possibly contributed to genetic risk for schizophrenia in a higher genetic-loading sample, and the associated locus was not the Val ${ }^{108 / 158}$ Met variant but other variants $3^{\prime}$ to this region. The true functional variants associated with schizophrenia in our studies remain to be clarified. It will be worthwhile to map the 3' region of the COMT gene in a larger Asian family study, and to find the ethnicity-specific functional variants that contribute to the genetic association with schizophrenia and its influence on the function of the COMT gene. 


\section{Acknowledgments}

We acknowledge the help we received from the Department of Medical Research in National Taiwan University Hospital. This study was supported by grants from the National Science Council, Taiwan (NSC-91-3112-B-002-011; NSC-92-3112-B-002-019; NSC-93-3112-B-002-012; NSC-94-3112-B-002-020; NSC-95-3112-B-002011; NSC-96-3112-B-002-011), and the National Health Research Institute, Taiwan (NHRI-908825PP; NHRI-EX91，92, 93, 94-9113PP). The authors declare no conflicts of interest that could have influenced the reporting of these results.

\section{References}

1. McGue M, Gottesman II, Rao DC. The transmission of schizophrenia under a multifactorial threshold model. Am J Hum Genet 1983;35:1161-78.

2. Faraone SV, Tsuang D, Tsuang MT. Genetics of Mental Disorders: A Guide for Students, Clinicians, and Researchers. New York: Guilford, 1999.

3. Kelly BD. Schizophrenia and velo-cardio-facial syndrome. Lancet 2002;360:721-2.

4. Egan MF, Goldberg TE, Kolachana BS, et al. Effect of COMT Val108/158 Met genotype on frontal lobe function and risk for schizophrenia. Proc Natl Acad Sci USA 2001;98:6917-22.

5. Joober R, Gauthier J, Lal S, et al. Catechol-O-methyltransferase Val-108/158-Met gene variants associated with performance on the Wisconsin Card Sorting Test. Arch Gen Psychiatr 2002;59:662-3.

6. Diaz-Asper CM, Goldberg TE, Kolachana BS, et al. Genetic variation in catechol-O-methyltransferase: effects on working memory in schizophrenic patients, their siblings, and healthy controls. Biol Psychiatry 2008;63:72-9.

7. Meyer-Lindenberg A, Nichols T, Callicott JH, et al. Impact of complex genetic variation in COMT on human brain function. Mol Psychiatry 2006;11:797, 867-77.

8. Munafo MR, Bowes L, Clark TG, et al. Lack of association of the COMT (Val158/108 Met) gene and schizophrenia: a meta-analysis of case-control studies. Mol Psychiatry 2005;10:765-70.

9. Fan JB, Zhang CS, Gu NF, et al. Catechol-O-methyltransferase gene $\mathrm{Val} / \mathrm{Met}$ functional polymorphism and risk of schizophrenia: a large-scale association study plus metaanalysis. Biol Psychiatry 2005;57:139-44.

10. Shifman S, Bronstein $M$, Sternfeld $M$, et al. A highly significant association between a COMT haplotype and schizophrenia. Am J Hum Genet 2002;71:1296-302.
11. Bray NJ, Buckland PR, Williams NM, et al. A haplotype implicated in schizophrenia susceptibility is associated with reduced COMT expression in human brain. $\mathrm{Am} J$ Hum Genet 2003;73:152-61.

12. Dempster EL, Mill J, Craig IW, et al. The quantification of COMT mRNA in post mortem cerebellum tissue: diagnosis, genotype, methylation and expression. BMC Med Genet 2006;7:10

13. Chen X, Wang X, O'Neill AF, et al. Variants in the catecholo-methyltransferase (COMT) gene are associated with schizophrenia in Irish high-density families. Mol Psychiatry 2004;9:962-7.

14. Handoko HY, Nyholt DR, Hayward NK, et al. Separate and interacting effects within the catechol-O-methyltransferase (COMT) are associated with schizophrenia. Mol Psychiatry 2005;10:589-97.

15. Glatt SJ, Faraone SV, Tsuang MT. Association between a functional catechol O-methyltransferase gene polymorphism and schizophrenia: meta-analysis of case-control and familybased studies. Am J Psychiatry 2003;160:469-76.

16. Liou YJ, Tsai SJ, Hong CJ, et al. Association analysis of a functional catechol-o-methyltransferase gene polymorphism in schizophrenic patients in Taiwan. Neuropsychobiology 2001;43:11-4.

17. Nunokawa A, Watanabe $Y$, Muratake T, et al. No associations exist between five functional polymorphisms in the catechol-O-methyltransferase gene and schizophrenia in a Japanese population. Neurosci Res 2007;58:291-6.

18. Fan JB, Chen WY, Tang JX, et al. Family-based association studies of COMT gene polymorphisms and schizophrenia in the Chinese population. Mol Psychiatry 2002;7:446-7.

19. Chen $\mathrm{CH}$, Lee $\mathrm{YR}$, Chung MY, et al. Systematic mutation analysis of the catechol O-methyltransferase gene as a candidate gene for schizophrenia. Am J Psychiatry 1999;156: 1273-5.

20. Kay SR, Fiszbein A, Opler LA. The positive and negative syndrome scale (PANSS) for schizophrenia. Schizophr Bull 1987;13:261-76.

21. Hwu HG. Psychiatric Diagnostic the Assessment. Taipei: Publication Committee, College of Medicine, National Taiwan University, 1999:7-42.

22. Andreasen NC. The Scale for the Assessment of Negative Symptoms (SANS). lowa City, IA: University of lowa, 1983.

23. Andreasen NC. The Scale for the Assessment of Positive Symptoms (SAPS). lowa City, IA: University of lowa, 1984.

24. Chen WJ, Hsiao CK, Hsiao LL, et al. Performance of the Continuous Performance Test among community samples. Schizophr Bull 1998;24:163-74.

25. Nuechterlein KH. Vigilance in schizophrenia and related disorders. In: Steinhauer SR, Gruzelier JH, Zubin J, eds. Handbook of Schizophrenia. Volume 5: Neuropsychology, Psychophysiology and Information Processing. Amsterdam: Elsevier, 1991:397-433.

26. Tien AY, Spevack TV, Jones DW, et al. Computerized Wisconsin Card Sorting Test: comparison with manual administration. Kaohsiung J Med Sci 1996;12:479-85. 
27. Lin CC, Chen WJ, Yang HJ, et al. Performance on the Wisconsin Card Sorting Test among adolescents in Taiwan: norms, factorial structure, and relation to schizotypy. J Clin Exp Neuropsychol 2000;22:69-79.

28. Heaton RK, Chelune GI, Talley JL, et al. Wisconsin Card Sorting Test Manual: Revised and Expanded. Odessa, FL: Psychological Assessment Resources, 1993.

29. Goldberg TE, Weinberger DR, Berman KF, et al. Further evidence for dementia of the prefrontal type in schizophrenia? A controlled study of teaching the Wisconsin Card Sorting Test. Arch Gen Psychiatry 1987;44:1008-14.

30. Koren D, Seidman LJ, Harrison RH, et al. Factor structure of the Wisconsin Card Sorting Test: dimensions of deficit in schizophrenia. Neuropsychology 1998;12:289-302.

31. Wolf LE, Cornblatt BA, Roberts SA, et al. Wisconsin Card Sorting deficits in the offspring of schizophrenics in the New York High-Risk Project. Schizophr Res 2002;57:173.

32. O'Connell JR, Weeks DE. PedCheck: a program for identification of genotype incompatibilities in linkage analysis. Am J Hum Genet 1998;63:259-66.

33. Terwilliger JDaO. Handbook of Human Genetic Linkage. Baltimore: Johns Hopkins University Press, 1994.

34. Clayton D. A generalization of the transmission/disequilibrium test for uncertain-haplotype transmission. Am J Hum Genet 1999;65:1170-7.

35. Horvath S, Xu X, Laird NM. The family based association test method: strategies for studying general genotypephenotype associations. Eur J Hum Genet 2001;9:301-6.

36. Abecasis GR, Cookson WO. GOLD-graphical overview of linkage disequilibrium. Bioinformatics 2000;16:182-3.

37. Tsai SJ, Hong CJ, Liao DL, et al. Association study of a functional catechol-O-methyltransferase genetic polymorphism with age of onset, cognitive function, symptomatology and prognosis in chronic schizophrenia. Neuropsychobiology 2004;49:196-200.

38. Park TW, Yoon KS, Kim JH, et al. Functional catechol-Omethyltransferase gene polymorphism and susceptibility to schizophrenia. Eur Neuropsychopharmacol 2002;12: 299-303.

39. Yu R, Zhang XN, Huang XX, et al. Association analysis of COMT polymorphisms and schizophrenia in a Chinese
Han population: a case-control study. Am J Med Genet B Neuropsychiatr Genet 2007;144B:570-3.

40. Palmatier MA, Pakstis AJ, Speed W, et al. COMT haplotypes suggest P2 promoter region relevance for schizophrenia. Mol Psychiatry 2004;9:859-70.

41. Lee SG, Joo Y, Kim B, et al. Association of Ala72Ser polymorphism with COMT enzyme activity and the risk of schizophrenia in Koreans. Hum Genet 2005;116:319-28.

42. Bruder GE, Keilp JG, Xu H, et al. Catechol-O-methyltransferase (COMT) genotypes and working memory: associations with differing cognitive operations. Biol Psychiatry 2005;58:901-7.

43. Bilder RM, Volavka J, Czobor P, et al. Neurocognitive correlates of the COMT Val(158)Met polymorphism in chronic schizophrenia. Biol Psychiatry 2002;52:701-7.

44. Chan RC, Chen RY, Chen EY, et al. The differential clinical and neurocognitive profiles of COMT SNP rs165599 genotypes in schizophrenia. J Int Neuropsychol Soc 2005;11: 202-4.

45. Pelayo-Teran JM, Crespo-Facorro B, Carrasco-Marin E, et al. Catechol-O-methyltransferase Val158Met polymorphism and clinical characteristics in first episode nonaffective psychosis. Am J Med Genet B Neuropsychiatr Genet 2008;147B:550-6.

46. Strous RD, Lapidus R, Viglin D, et al. Analysis of an association between the COMT polymorphism and clinical symptomatology in schizophrenia. Neurosci Lett 2006; 393:170-3.

47. Laruelle M, Kegeles LS, Abi-Dargham A. Glutamate, dopamine, and schizophrenia: from pathophysiology to treatment. Ann N Y Acad Sci 2003;1003:138-58.

48. Petronis A, Sherrington RP, Paterson AD, et al. Genetic anticipation in schizophrenia: pro and con. Clin Neurosci 1995;3:76-80.

49. Borrmann-Hassenbach MB, Albus M, Scherer J, et al. Age at onset anticipation in familial schizophrenia. Does the phenomenon even exist? Schizophr Res 1999;40:55-65.

50. Kendler KS, Karkowski-Shuman L, Walsh D. Age at onset in schizophrenia and risk of illness in relatives. Results from the Roscommon Family Study. $\mathrm{Br} /$ Psychiatry 1996;169:213-8. 